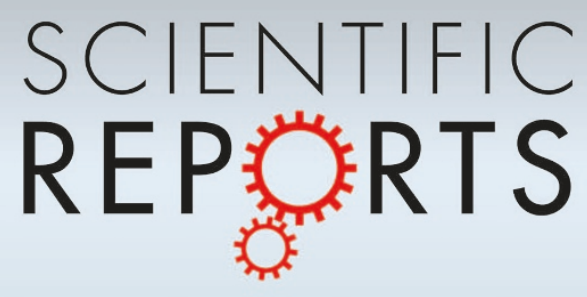

\title{
OPEN A critical re-assessment of DNA repair gene promoter methylation in non-small cell lung carcinoma
}

SUBJECT AREAS:

DNA METHYLATION

BIOMARKER RESEARCH

PREDICTIVE MARKERS

NON-SMALL-CELL LUNG CANCER

Received

17 July 2013

Accepted

13 January 2014

Published

26 February 2014

\author{
Hongdo Do ${ }^{1,2,6}$, Nicholas C. Wong ${ }^{2,6}$, Carmel Murone ${ }^{2}$, Thomas John ${ }^{3}$, Benjamin Solomon ${ }^{4,5}$, \\ Paul L. Mitchell ${ }^{3}$ \& Alexander Dobrovic ${ }^{1,2,4,5}$
}

\begin{abstract}
'Molecular Pathology Research and Development Laboratory, Department of Pathology, Peter MacCallum Cancer Centre, Melbourne, Victoria, 8006, Australia, ${ }^{2}$ Translational Genomics and Epigenomics Laboratory, Ludwig Institute for Medical Research, Olivia Newton-John Cancer and Wellness Centre, Austin Hospital, Heidelberg, Victoria, 3084, Australia, ${ }^{3}$ Joint Austin-Ludwig Medical Oncology Department, Olivia Newton-John Cancer and Wellness Centre, Austin Hospital, Heidelberg, Victoria, 3084, Australia, ${ }^{4}$ Sir Peter MacCallum Department of Oncology, University of Melbourne, Parkville, Victoria, 3010, Australia, ${ }^{5}$ Division of Cancer Medicine, Peter MacCallum Cancer Centre, Melbourne, Victoria, 8006, Australia, ${ }^{6}$ Department of Pathology, University of Melbourne, Parkville, Victoria, 3010, Australia.
\end{abstract}

Correspondence and requests for materials should be addressed to A.D. (alex.dobrovic@ ludwig.edu.au)

DNA repair genes that have been inactivated by promoter methylation offer potential therapeutic targets either by targeting the specific repair deficiency, or by synthetic lethal approaches. This study evaluated promoter methylation status for eight selected DNA repair genes (ATM, BRCA1, ERCC1, MGMT, MLH1, NEIL1, RAD23B and XPC) in 56 non-small cell lung cancer (NSCLC) tumours and 11 lung cell lines using the methylation-sensitive high resolution melting (MS-HRM) methodology. Frequent methylation in NEIL1 (42\%) and infrequent methylation in ERCC1 (2\%) and RAD23B (2\%) are reported for the first time in NSCLC. MGMT methylation was detected in 13\% of the NSCLCs. Contrary to previous studies, methylation was not detected in $A T M, B R C A 1, M L H 1$ and XPC. Data from The Cancer Genome Atlas (TCGA) was consistent with these findings. The study emphasises the importance of using appropriate methodology for accurate assessment of promoter methylation.

$\mathrm{T}$ here is accumulating evidence that tumour response to DNA-damaging agents is associated with the expression levels of DNA repair genes. BRCA1 mRNA expression has shown to be associated with cisplatin resistance ${ }^{1-3}$ and docetaxel sensitivity ${ }^{4,5}$ in breast, ovarian, lung and gastric cancers. Tailored chemotherapy based on BRCA1 mRNA levels has also shown to improve patient survival in lung ${ }^{2,6}$, bladder ${ }^{7}$ and ovarian cancers $^{8}$. Similarly, high ERCC1 and MGMT mRNA expression has shown to confer resistance to platinum compounds ${ }^{9}$ and sensitivity to alkylating agents ${ }^{10,11}$. Expression profiling of selected DNA repair genes thus has potential for the better stratification of cancer patients who are likely to respond to DNA-damaging agents ${ }^{12}$.

Formalin-fixed paraffin-embedded (FFPE) tissues are commonly the only available clinical material for diagnostic analysis and for cancer biomarker studies. When FFPE tissue is used, expression profiling of DNA repair genes is often limited by low quantity and degraded RNA due to the detrimental effects of formalin fixation on nucleic acids. Moreover, normal cell contamination that is almost inevitably occurring during RNA extraction, even after macrodissection of tumour-enriched regions, confounds the interpretation of gene expression data.

Promoter methylation is an epigenetic mechanism leading to transcriptional silencing of gene expression and thus has been used for indirect assessment of gene expression. The use of relatively stable DNA for testing of molecular biomarkers provides an important advantage over RNA-based testing with respect to extraction, handling and storage conditions ${ }^{13}$. In human cancers, a number of DNA repair genes have been shown to undergo transcriptional silencing by DNA methylation ${ }^{14}$.

Previous studies have reported the occurrence of promoter methylation in several DNA repair genes in nonsmall cell lung cancer (NSCLC). However, the reported frequency of promoter methylation in NSCLC varies markedly between studies; ATM (0-47\%), BRCA1 (4-30\%), MGMT (8-50\%) and MLH1 (0-68\%) (Table 1). This high variation reflects the different methodologies used and emphasises that the DNA methylation status of clinically relevant genes needs to be validated using reliable and reproducible methodology before testing for their methylation has any clinical validity. 


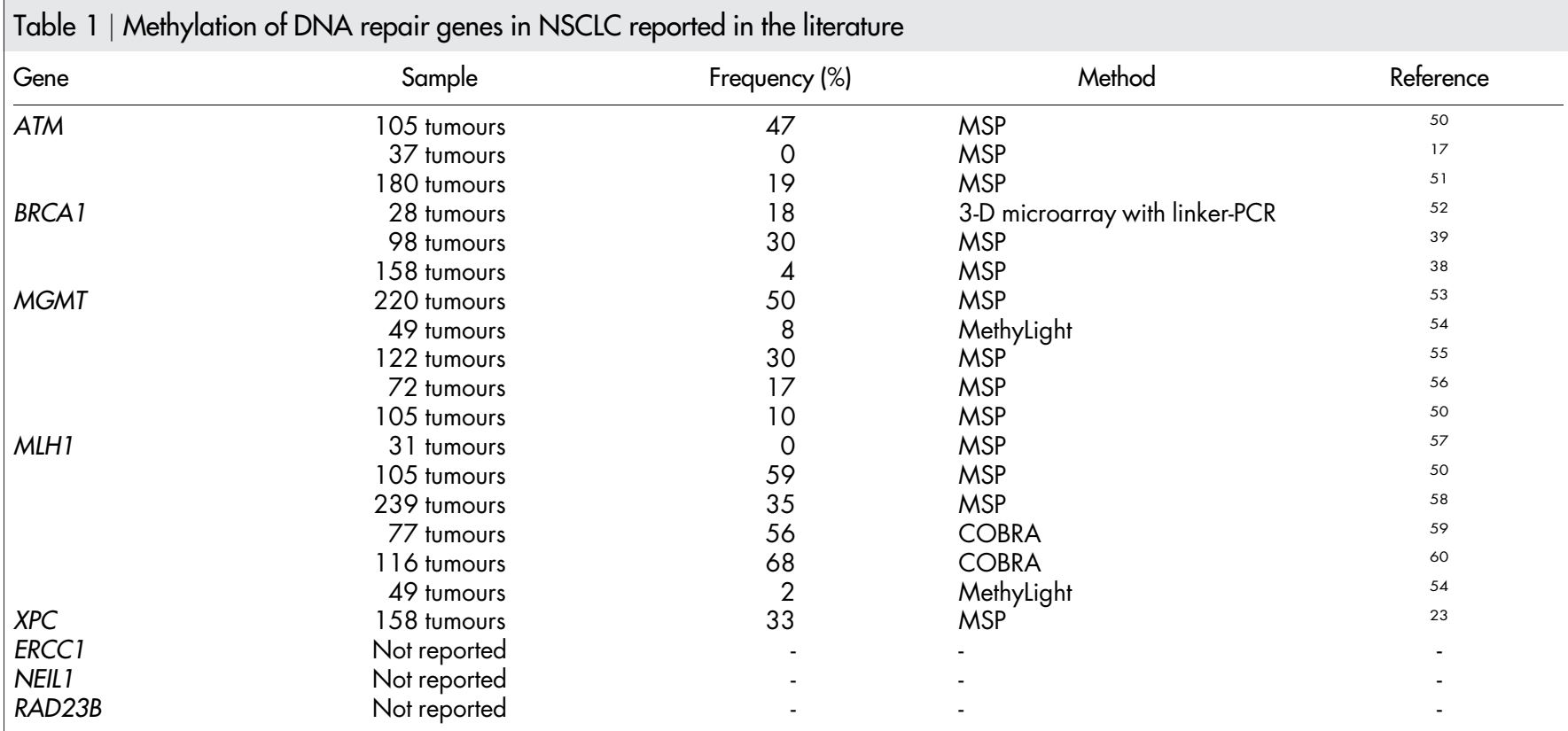

It is important to note that most of the previous studies used methylation-specific PCR (MSP), a method that has multiple limitations ${ }^{15,16}$. Detection of methylation status by MSP is based on endpoint analysis of PCR amplification by gel electrophoresis, which only provides qualitative results. As a consequence, the level of methylation and the pattern of methylation (homogeneous or heterogeneous) cannot be assessed. Furthermore, false positive results can be generated depending on primer design and the stringency of the assay conditions ${ }^{17}$.

It is thus important to critically re-evaluate previous methylation reports using methodologies with a low risk of false positives. In this study, we used methylation sensitive-high resolution melting (MSHRM) methodology for detection of promoter methylation. MSHRM is a sensitive and closed-tube methodology developed for detection of methylation in a locus-specific manner, utilizing the different melting temperatures $(\mathrm{Tm})$ of methylated and unmethylated DNA after bisulfite modification ${ }^{18}$. Due to the high Tm difference between methylated and unmethylated DNA, methylated samples are readily identifiable by analysing melting profiles. MSHRM allows semi-quantitative assessment of methylation levels when all the examined $\mathrm{CpG}$ sites are methylated, but like other methods cannot quantify when the methylation is heterogeneous ${ }^{16}$.

The aim of this study was to assess the promoter region methylation status of DNA repair genes of potential clinical importance. One set of genes had been previously investigated in NSCLC (ATM, BRCA1, MLH1, MGMT and XPC). We also assessed two DNA repair genes that had been reported as methylated in other tumour types (NEIL1 and RAD23B), and a gene (ERCC1) whose gene expression levels had been reported as having predictive significance in NSCLC ${ }^{19}$.

\section{Results}

Re-assessing the methylation status of DNA repair genes reported to be methylated in NSCLC. DNA methylation of the promoter region CpG islands in the ATM, BRCA1, MGMT, MLH1 and XPC genes has been previously reported in lung cancer. We assessed the methylation status of these CpG islands in 11 lung cancer cell lines and 56 NSCLC tumours using methylation sensitive-high resolution melting (MS- HRM). The MS-HRM assay conditions for ATM, $B R C A 1, M G M T$ and $M L H 1$ were robust and published in our previous studies ${ }^{18,20-22}$. We designed our ERCC1 and XPC MS-
HRM assay to screen the region where methylation was reported in a previous study ${ }^{23}$. A series of dilutions of methylated DNA in unmethylated DNA $(100 \%, 50 \%, 25 \%, 10 \%, 5 \%, 1 \%, 0 \%)$ were used in each MS-HRM run as controls.

We did not find promoter methylation in four of these five DNA repair genes (ATM, BRCA1, MLH1 and XPC) in any of the 11 lung cancer cell lines (Table 2) or in the 56 NSCLC tumours (Figure 1). Although XPC methylation was previously reported in the H1355 and Calu-1 cell lines, we did not find any XPC methylation in these cell lines (Figure 1). All methylated controls tested in each MS-HRM run were readily interpreted as methylated as their melting profiles differed significantly from that of unmethylated controls.

MGMT methylation was found in three lung cancer cell lines (H69, H1666, and H1755) (Table 2) and seven of the 56 NSCLC tumours (13\%) (Figure 2. Panel A). The melting patterns of the methylated lung cancer cell lines and NSCLC tumours were indicative of heterogeneous methylation i.e. the methylation status of individual CpGs varied across the amplicon. The level of heterogeneous methylation cannot be readily estimated by visual examination of the melting curves as the methylation dilution controls are only useful for estimation of methylated alleles of homogeneously methylated samples. However, the melting profiles of the lung cancer cell lines were indicative of low (H69, H1975) and moderate (H1666) numbers of methylated cytosines in the interrogated region. Similarly, only low or moderate levels of heterogeneous MGMT methylation were detected in seven NSCLC tumours.

Assessing the methylation status of DNA repair genes not previously tested in NSCLC. We also assessed the promoter methylation of NEIL1, ERCC1 and RAD23B as their methylation has been reported in other cancers, but has not previously been examined in NSCLC $^{24-26}$.

Frequent NEIL1 methylation was found in lung cancer cell lines and in NSCLC tumours. In the lung cancer cell lines, high levels of NEIL1 methylation were detected in H460 (100\%) and H1355 (nearly 100\%). The other cell lines showed heterogeneous methylation either at moderate levels (H1650, H2087 and H1975) or at low levels (H1395, H1755, H1666, H69, H2228, and Calu-1). Bisulfite Sanger sequencing of selected lung cancer cell lines confirmed the presence of NEIL1 methylation. NEIL1 methylation was also detected in 23 of the 56 NSCLC tumours (42\%). All methylated 


\begin{tabular}{|c|c|c|c|c|c|c|c|c|c|c|}
\hline \multirow[b]{2}{*}{ Cell line } & \multicolumn{2}{|c|}{ Control genes } & \multicolumn{8}{|c|}{ DNA repair genes } \\
\hline & APC & CDKN2A & ATM & BRCA 1 & ERCC 1 & MGMT & MLHI & NEIL1 & RAD23B & XPC \\
\hline H1395 & UM & UM & UM & UM & UM & UM & UM & Low & UM & UM \\
\hline $\mathrm{H} 1650$ & $100 \%$ & NA & UM & UM & UM & UM & UM & Moderate & UM & UM \\
\hline $\mathrm{H} 460$ & $50 \%$ & NA & UM & UM & UM & UM & UM & $100 \%$ & UM & UM \\
\hline $\mathrm{H} 1755$ & UM & NA & UM & UM & UM & Low & UM & Low & UM & UM \\
\hline H2O87 & $50 \%$ & UM & UM & UM & Low & UM & UM & Moderate & UM & UM \\
\hline $\mathrm{H} 2228$ & $5 \%$ & NA & UM & UM & UM & UM & UM & Low & UM & UM \\
\hline H1975 & UM & UM & UM & UM & UM & UM & UM & Moderate & UM & UM \\
\hline H1355 & $100 \%$ & $100 \%$ & UM & UM & UM & UM & UM & $\sim 100 \%$ & UM & UM \\
\hline Calu-1 & UM & $100 \%$ & UM & UM & UM & UM & UM & Low & UM & UM \\
\hline
\end{tabular}

NSCLC tumours showed heterogeneous methylation pattern either at moderate levels (13 samples) or at low levels (10 samples) (Figure 2. Panel B).

Methylation in ERCC1 and RAD23B was rarely detected. ERCC1 methylation was found in $\mathrm{H} 2087$ and one of the NSCLC tumours, the former showing a low-level heterogeneous methylation pattern and the latter showing a moderate-level heterogeneous methylation pattern. RAD23B methylation was detected in one NSCLC tumour and was not found in any of the lung cancer cell lines. Bisulfite Sanger sequencing of the tumour samples confirmed the methylation status (Figure 2. Panels C and D).

Methylation status of two control genes using MS-HRM. Given the low frequency of methylation of our target DNA repair gene set, we also assessed the promoter methylation status of the $A P C$ and $C D K N 2 A$ genes in the cell lines and tumours to confirm that neither the bisulfite modification protocol nor the MS-HRM analysis precluded the detection of promoter methylation. The APC and $C D K N 2 A$ genes were chosen as a relatively high methylation frequency for each has been previously found by multiple laboratories ${ }^{27-29}$. A high proportion of samples were methylated for either one or both of the genes, indicating that methylation, where present, could be readily identified by our MS-HRM assays.

$A P C$ methylation was found in five lung cancer cell lines (H460, H1355, H1650, H2087, and H2228) (Figure 3. Panel A). The melting patterns of all methylated lung cancer cell lines were suggestive of homogeneous methylation. By comparing with methylation dilution controls, the level of APC methylation was estimated to be $100 \%$ in
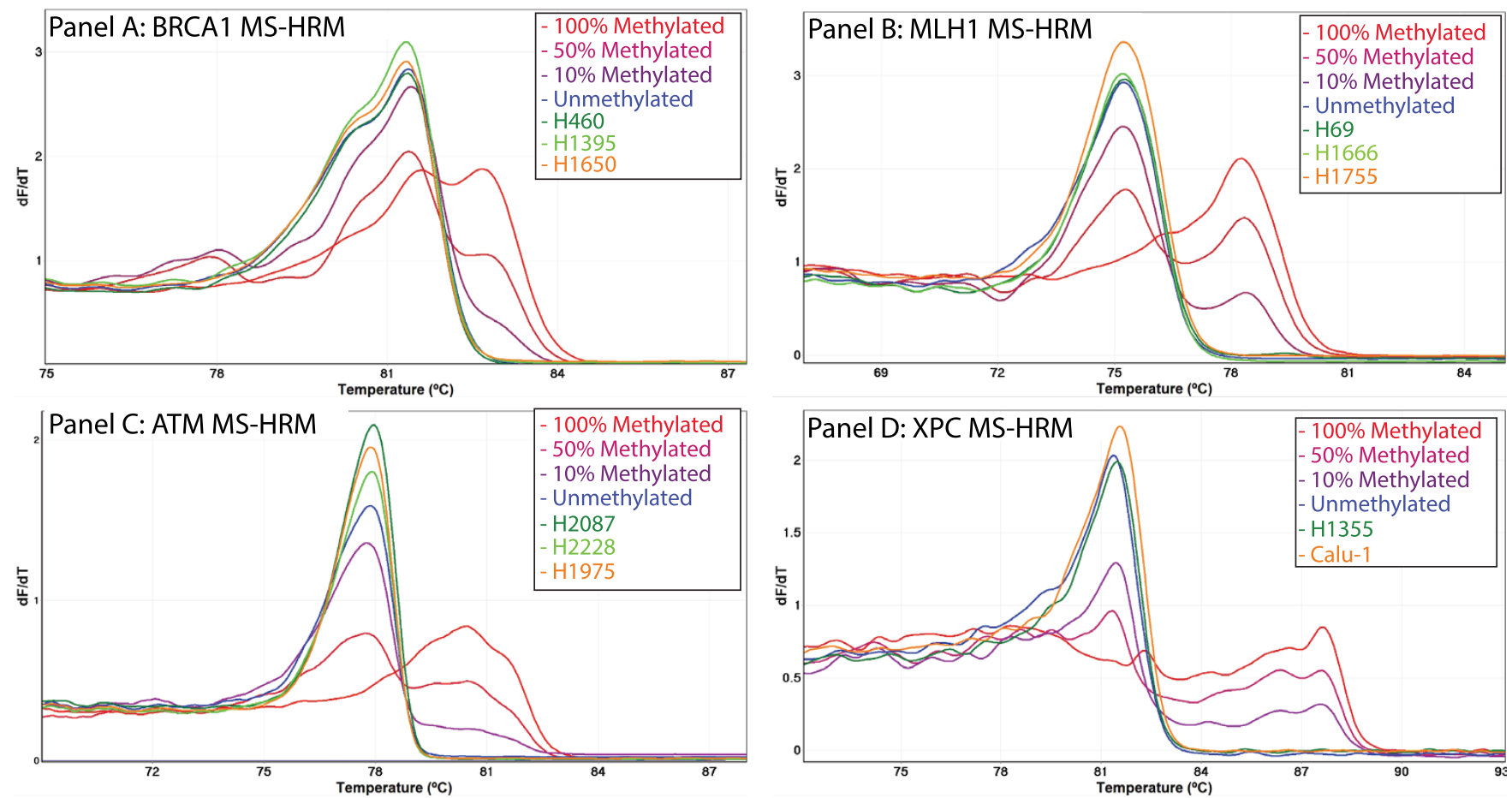

Figure $1 \mid$ Absence of ATM, BRCA1, MLH1 and XPC methylation in lung cancer cell lines and tumours. DNA methylation in the promoter regions of the ATM, BRCA1, MLH1 and XPC genes was assessed in 11 lung cancer cell lines and 56 NSCLC tumours using MS-HRM. After bisulfite modification, methylated DNA that retains cytosines has a higher melting temperature compared with unmethylated DNA that contains thymine (uracil before PCR). All samples having different melting patterns compared with unmethylated DNA control (in blue) are considered as methylated. Promoter methylation in $A T M, B R C A 1, M L H 1$ and XPC was not detected in any of the lung cancer cell lines and the NSCLC tumours. The negative first derivative plot of three representative lung cancer cell line samples are shown for BRCA1 (Panel A), MLH1 (Panel B), ATM (Panel C) and XPC (Panel D). Absence of XPC methylation is seen for the two lung cancer cell lines (H1355 and Calu-1) that were previously reported to be methylated. 

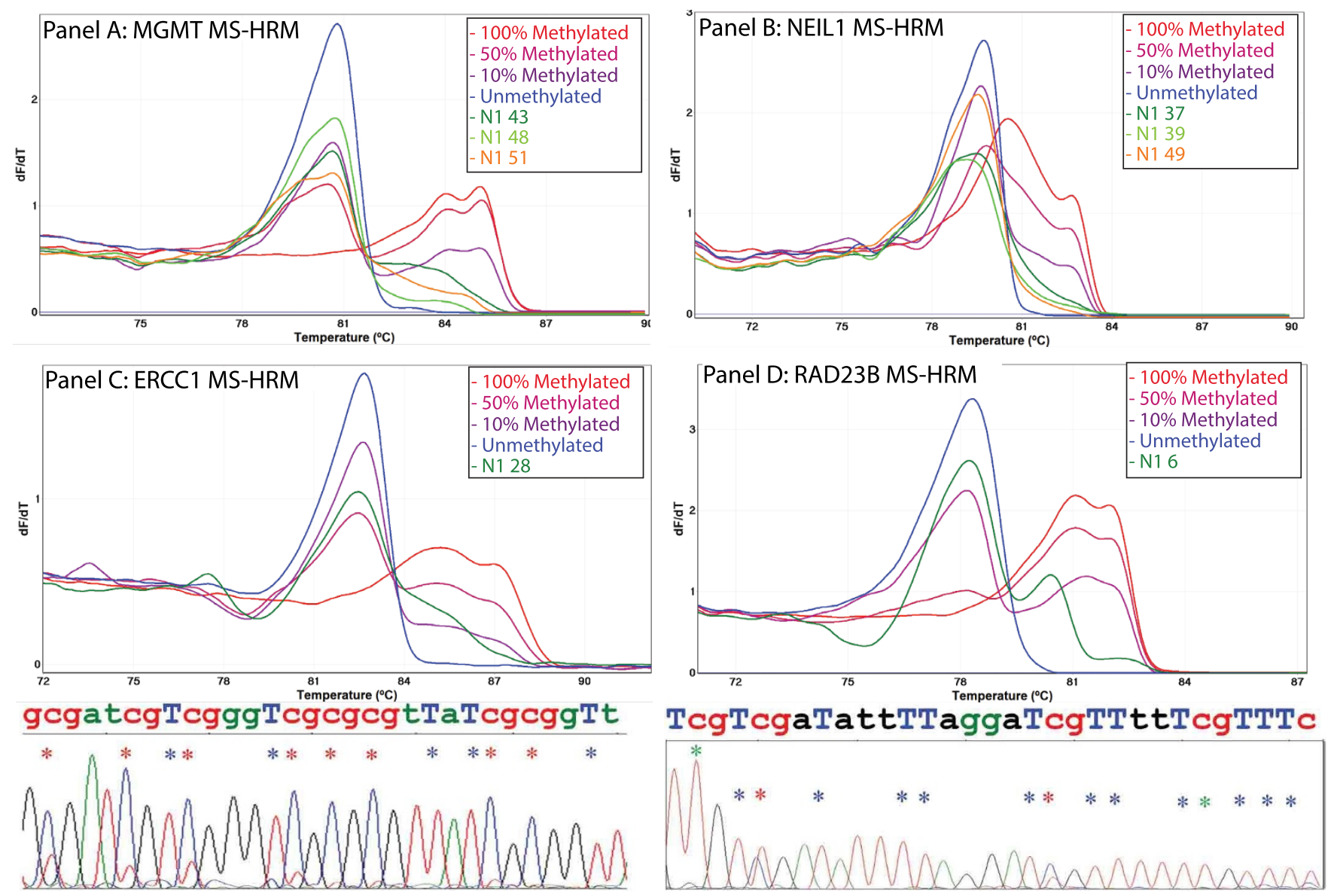

Figure $2 \mid$ Detection of methylation in MGMT, NEIL1, ERCC1 and RAD23B in NSCLC tumours. DNA methylation in the promoter regions of the MGMT, NEIL1, ERCC1 and RAD23B genes was assessed in 56 NSCLC tumours using MS-HRM. Methylation was detected in MGMT (25\%), NEIL1 (42\%), ERCC1 (2\%) and RAD23B (2\%). The negative first derivative plots of representative methylated NSCLC tumours for MGMT(Panel A) and NEIL1 (Panel B), ERCC1 (Panel C) and RAD23B (Panel D) are shown. Bisulfite Sanger sequencing traces confirming the ERCC1 and RAD23B methylation status are shown below the negative first derivative plots. Red, green and blue asterisks indicate the positions of methylated cytosines, unmethylated cytosines and bisulfite conversion controls respectively.

$\mathrm{H} 1650$ and $\mathrm{H} 1355$, and 50\% in $\mathrm{H} 460$ and $\mathrm{H} 2987$. Interestingly, APC methylation was estimated to be about $5-10 \%$ in $\mathrm{H} 2228$, suggestive of differential APC methylation status within the $\mathrm{H} 2228$ cells.

Homogenous CDKN2A methylation was found in two lung cancer cell lines (H1355 and Calu-1) and was estimated to be 100\% (Figure 3. Panel B). Five of the lung cancer cell lines (H460, $\mathrm{H} 1650, \mathrm{H} 1666, \mathrm{H} 1755$, and H2228), that were previously found to have a homozygous $C D K N 2 A$ deletion (CONAN database), were not amplified by the CDKN2A MS-HRM assay, confirming the absence of CDKN2A template. Four cell lines (H1395, H69, H2987 and H1975) were negative for CDKN2A methylation.

We also tested the 56 NSCLC tumours for methylation in the APC and $C D K N 2 A$ promoter regions. $A P C$ and $C D K N 2 A$ methylation was detected in 14 NSCLC tumours each (25\%) (Figure 3. Panels C and D). A total of 23 NSCLC tumours (41\%) were methylated for at least one of the genes and methylation in both genes was detected in 5 NSCLC tumours.

External validation using The Cancer Genome Atlas (TCGA) database. As we observed a considerable discrepancy in the methylation frequency of tested DNA repair genes between our MS-HRM results and the literature, we sought to validate our results using a second dataset generated by another methodology with a low false positive rate. We analysed the methylation data from the TCGA NSCLC database that provides genome-wide methylation status assessed by the HumanMethylation $450 \mathrm{k}$ beadchip (Illumina).
Methylation data were available from 568 NSCLC tumours, comprising of 341 adenocarcinomas and 227 squamous cell carcinomas.

We searched for HM450k probes overlapping the CpG sites within our eight MS- HRM amplicons. No overlapping CpG sites were detected for ATM, ERCC1 and MGMT. Eight probes were identified for the remaining genes; two probes for $B R C A 1$, one for $M L H 1$, one for NEIL1, three for RAD23B and one for XPC (Figure 4).

Methylation was completely absent in the three CpG sites of $R A D 23 B$ and the CpG site of XPC in the 568 NSCLC samples. Very low frequency methylation was seen for $M L H 1$ (3/567, 0.5\%). Three primary squamous cell carcinomas of lung showed methylation at both CpG sites of BRCA1 (3/568, 0.5\%) although one of the sites in the third sample was just below the $20 \%$ cut-off. These results were consistent with our MS-HRM results finding either absent or very rare methylation in the BRCA1, MLH1, RAD23B and XPC promoters.

The CpG site in the NEIL1 promoter was frequently methylated (478/568, 84\%), although the level of methylation varied among the methylated samples. This TCGA methylation data again confirmed our MS-HRM results demonstrating high frequency NEIL1 methylation in NSCLC.

\section{Discussion}

Epigenetic alterations in cancer are a potential source of therapeutic targets for personalised cancer treatment. MGMT methylation in glioma is the best known example where it predicts a durable res- 

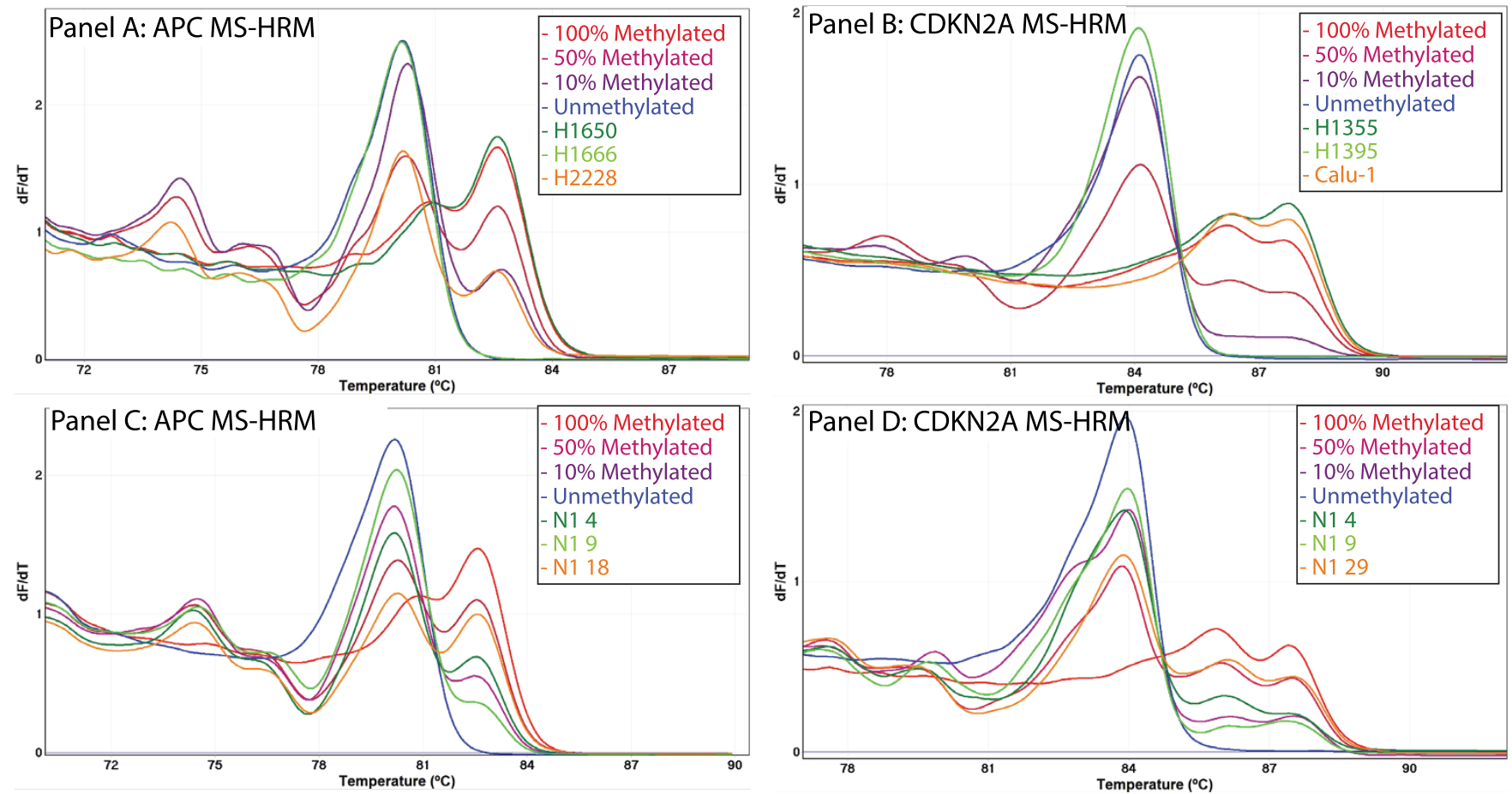

Figure $3 \mid$ Assessment of methylation status for APC and CDKN2A in lung cancer cell lines and tumours using MS-HRM. DNA methylation in the promoter regions of the APC and CDKN2A genes was assessed in 11 lung cancer cell lines and 56 NSCLC tumours using MS-HRM. APC was found in 5 lung cancer cell lines and 14 NSCLC tumours. CDKN2A methylation was detected in 2 lung cancer cell lines and 14 NSCLC tumours. The negative first derivative plots of three representative methylated lung cancer cell lines for APC (Panel A) and CDKN2A (Panel B) and three representative methylated NSCLC tumours for APC (Panel C) and CDKN2A (Panel D) are shown.

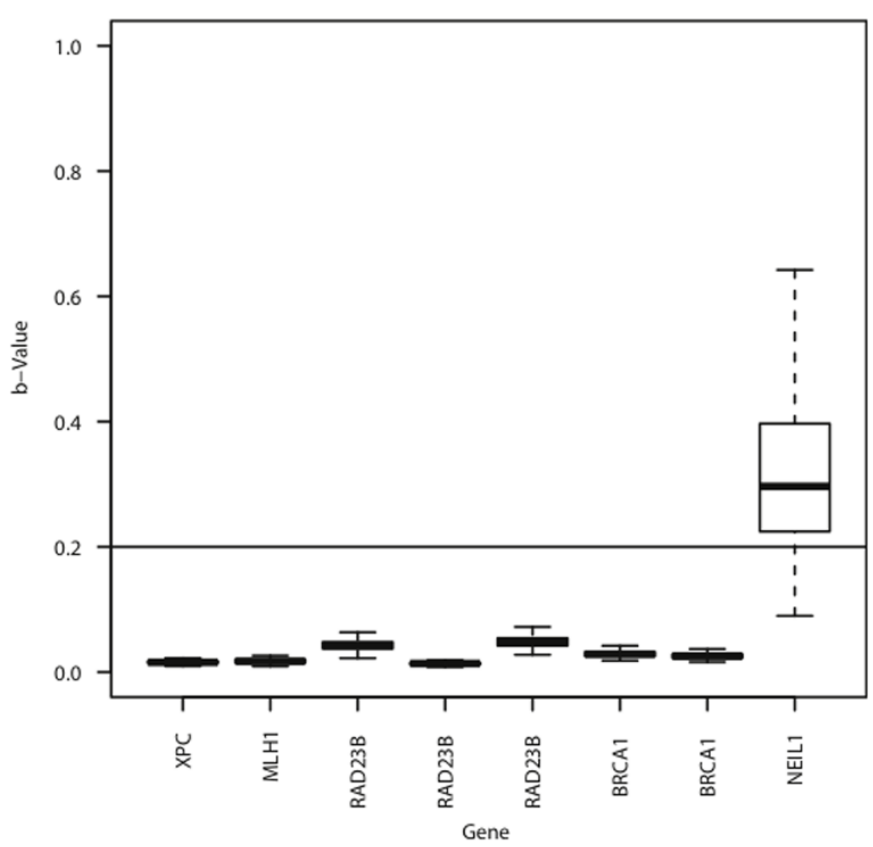

Figure $4 \mid$ The TCGA methylation data of five DNA repair genes. TCGA methylation data from 568 non-small cell lung cancers for the eight overlapping CpG sites with our MS-HRM amplicons is presented as boxplots. Two overlapping CpG sites for $B R C A 1$, one for $M L H 1$, one for $N E I L 1$, three for RAD23B and one for XPC were analysed. A $\beta$-value of greater than 0.2 was used to define the presence of DNA methylation as shown by the horizontal line. Consistent with our MS-HRM results, absent or very rare methylation was found for BRCA1, MLH1, RAD23B and XPC, and highly frequent methylation was found for NEIL1. ponse to treatment with alkylating agents ${ }^{30}$. Methylation of other DNA repair genes also has been considered for the selection of optimal chemotherapeutic agents for the treatment of cancer, although these have not been clinically implemented up to now ${ }^{31}$. Before clinical implementation, individual methylation markers need to be rigorously validated, ideally by using different methodo$\operatorname{logies}^{13}$. In this study, we sought to validate a range of previously reported and to examine novel methylated DNA repair markers that could potentially be therapeutically exploited.

In the literature, highly variable estimates of the frequency of methylation for the ATM (0-47\%), BRCA1 (4-30\%), MLH1 (0$68 \%), M G M T(8-50 \%)$, and XPC (33\%) genes have been reported in NSCLC tumours (Table 1). As most of the previous studies used the MSP method to determine the methylation status of candidate genes, the previous findings needed to be validated using other methodologies that are less prone to give false results. When we assessed DNA methylation using MS-HRM, we did not find methylation in any of these four DNA repair genes (ATM, BRCA1, MLH1, and XPC) in our 11 lung cancer cell lines and 56 NSCLC samples. This was consistent with the TCGA data which showed either absent or very low frequency of methylation for these promoters.

There are several possible explanations for the discrepant results, including differences in ethnicity or clinicopathological features of samples tested in each study. Several studies have reported varying methylation frequencies in cancer between different ethnic groups, including in the promoters of the IGFBP $3^{32}, T M S 1^{33}$ and GSTP1 genes $^{34}$. However, the real reasons for the discrepant results are likely to be technical such as scoring of low-level methylation, false positives due to the use of inadequately designed primers or amplification of methylated pseudogene sequences.

Low levels of methylation, especially present at $\leq 1 \%$, can cause discrepant results due to the different analytic sensitivity of detection methods. The lower limit of MSP can be close to $0.1 \%{ }^{35}$, allowing samples with low level methylation to be interpreted as methylated. 
A high frequency of false positive ATM methylation calls deriving from the use of inadequate MSP conditions has been previously demonstrated in NSCLC $^{17}$. ATM methylation was not detected in NSCLC when strict guidelines for performance of MSP are used ${ }^{17}$. We did not find ATM methylation in our 11 lung cancer cell lines and 56 lung tumours, confirming the absence of ATM methylation.

The BRCA1 pseudogene (BRCA1P1), a duplicated region of $B R C A 1$ exons $1 \mathrm{~A}, 1 \mathrm{~B}$, and 2 , has a strong sequence homology to the $B R C A 1$ gene. As methylation of $B R C A 1 P 1$ has been previously reported in cancers ${ }^{36,37}$, there is a risk of false positives due to amplification of the methylated $B R C A 1 P 1$ sequence. Two previous studies have assessed the BRCA1 methylation status in NSCLC tumours using MSP as a detection method ${ }^{38,39}$. The MSP primers designed by Lee et al. have 19 (19/21 in the forward) and 17 (17/20 bases in the reverse) matched bases, including the nine consecutive bases from the $3^{\prime}$ end of both primers, to the BRCA1 pseudogene ${ }^{39}$. The frequency of $B R C A 1$ methylation (30\%) reported by Lee et al. was 7 -fold higher than that of being reported by Marsit et al. (4\%) where more stringent MSP primers were used to avoid the amplification of the methylated $B R C A 1 P 1$ sequence.

$X P C$ methylation was initially reported in NSCLC tumours and lung cancer cell lines ${ }^{23}$. Wu et al. reported that XPC methylation was detectable in $34 \%$ of NSCLC tumours by HpaII-based PCR and in four lung cancer cell lines harboring TP53 mutations. Surprisingly, XPC methylation was not detected in this cohort of 56 NSCLC tumours and in two of the lung cancer cell lines (H1355 and Calu1) previously reported as methylated ${ }^{23}$. The absence of XPC methylation was confirmed in the TCGA data. None of the 568 NSCLC tumours of the TCGA study had XPC methylation at the overlapping CpG site with our MS-HRM amplicon. As our XPC MS-HRM assay was designed to amplify those methylated $\mathrm{CpG}$ sites in the Wu et al. study, the discordant results was thus not likely to be caused by the examination of different $\mathrm{CpG}$ sites. To assess the XPC methylation status in NSCLC tumours, Wu et al. used the HpaII restriction endonuclease for selective cleavage of unmethylated DNA before PCR amplification $^{23}$. However, there is a risk of incomplete enzymatic digestion of unmethylated DNA by HpaII, potentially resulting in false positives ${ }^{40,41}$.
This study is the first report showing methylation of ERCC1, $R A D 23 B$ and NEIL1 in NSCLC. The excision repair cross-complementing group 1 (ERCC1) is a rate-limiting protein involved in the recognition and excision of DNA adducts. Recently, Chen et al. reported that ERCC1 methylation was significantly associated with chemosensitivity to cisplatin in glioma cell lines and glioma tumours $^{25}$. In NSCLC, low levels of ERCC1 expression were correlated with favorable clinical outcomes of prolonged survival and sensitivity to platinum-based chemotherapies ${ }^{19}$. Therefore, ERCC1 methylation may serve as a predictive biomarker for identification of NSCLC patients who are highly sensitive to platinum-based chemotherapies. These patients potentially would have a more durable response than patients with high levels of ERCC1 expression that were not methylated.

The RAD23B protein forms a DNA damage recognition complex with the XPC and centrin 2 proteins. The RAD23B/XPC/centrin 2 complex recognises and interacts with the damaged bases or the sugar-phosphate backbone of DNA in the NER pathway ${ }^{42}$. High RAD23B expression has been suggested as a promising biomarker associated with response to histone deacetylase (HDAC) inhibitors in cutaneous T-cell lymphoma patients ${ }^{43}$. As anti-tumour activities of HDAC inhibitors have been demonstrated in NSCLC ${ }^{44}$, it can be speculated that silencing of RAD23B expression through promoter methylation makes tumour cells more resistant to HDAC inhibitors. On the other hand, $R A D 23 B$ methylation may make tumour cells more sensitive to DNA-damaging agents.

Nei-like 1 (NEIL1), an ortholog of E.coli Nei (endonuclease VIII), is a bifunctional DNA glycosylase that repairs oxidative DNA damage of 8-hydroxyguanine and thymine glycol ${ }^{45}$ and protects cells from radiation-mediated cell death ${ }^{46}$. Recently, epigenetic silencing of NEIL1 through promoter methylation was reported in head and neck squamous cell carcinomas ${ }^{24}$. In this study, we found that the NEIL1 promoter is methylated in NSCLC at a high frequency (42\%). This may identify patients that are sensitive to radiotherapy which deserves further study.

In conclusion, this study showed that methylation frequency of $A T M, B R C A 1, M L H 1$ and XPC in NSCLC is likely to be overestimated in the literature, emphasising the importance of rigorous

Table 3 | Primer sequences and amplicon information for each MS-HRM assay

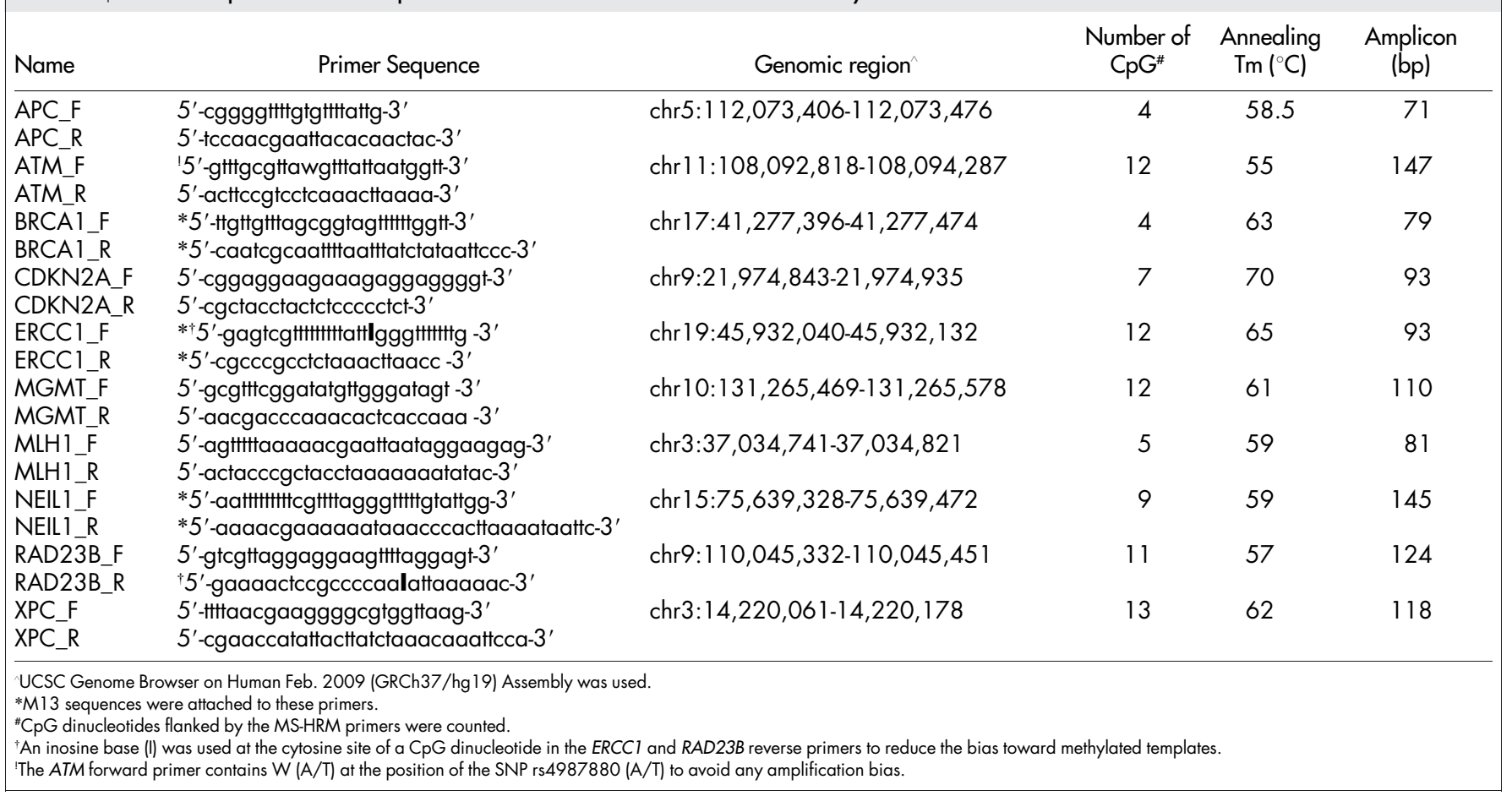


validation of previous data on DNA methylation. In particular, the use of adequate methodology is critical to avoid false positive results. DNA methylation in the ERCC1, RAD23B and especially the NEIL1 DNA repair genes may serve as useful biomarkers for the determination of molecularly tailored therapies in a subset of NSCLC patients.

\section{Methods}

Samples. Fifty-six N1 stage NSCLC tumours were collected at the Austin Hospital, Melbourne, Australia with the approval of the Austin Human Research Ethics Committee (project title and approval number "Biomarkers in the Australian Non Small Cell Lung Cancer Population" - H2006-02394). The methylation study was approved by the Ethics of Human Research Committee at the Peter MacCallum Cancer Centre, Melbourne, Australia (project title and approval number "Molecular Pathology of Cancer: Methylation, Mutation \& Expression" - 02/26).

DNA extraction from lung cancer cell lines. Lung cancer cell lines were cultured in RPMI 1640 medium with $25 \mathrm{mM}$ HEPES supplemented with $10 \%$ fetal bovine serum, and $0.1 \mathrm{units} / \mathrm{mL}$ of penicillin and $0.1 \mu \mathrm{g} / \mathrm{mL}$ of streptomycin. Cells were maintained at $37^{\circ} \mathrm{C}$ in a humidified chamber containing $5 \% \mathrm{CO} 2$. Cultured cells were harvested and washed twice with Dulbecco's phosphate-buffered saline buffer, followed by centrifugation at the speed of $10,000 \mathrm{rpm}$ for 10 minutes. Cell pellets were then suspended in $200 \mu \mathrm{L}$ of Dulbecco's phosphate-buffered saline buffer. After addition of $20 \mu \mathrm{L}$ of proteinase $\mathrm{K}(20 \mathrm{mg} / \mathrm{mL})$ and $200 \mu \mathrm{L}$ of buffer $\mathrm{AL}$, the suspended cells were incubating at $56^{\circ} \mathrm{C}$ for overnight. Genomic DNA was extracted from cultured lung cancer cell lines using the QIAamp DNA Blood Mini Kit (Qiagen, Hilden, Germany).

DNA extraction from NSCLC tumours. The tumour purity of individual NSCLC cases was assessed by pathologists at Peter MacCallum Cancer Centre and was estimated to be $40-95 \%$ with a median of $67 \%$. Two to five $5 \mu \mathrm{m}$ formalin-fixed tissue sections were washed with $1 \mathrm{~mL}$ of xylene to remove paraffin and were incubated at $40^{\circ} \mathrm{C}$ for 10 minutes. Supernatant was removed after a centrifugation at $13,000 \mathrm{rpm}$ for 10 minutes and tissue pellets were sequentially washed with $100 \%$ and $70 \%$ ethanol. The tissue pallets were then resuspended with $100 \mu \mathrm{L}$ of ATL buffer of the DNeasy Tissue kit (Qiagen) and incubated at $97^{\circ} \mathrm{C}$ for 15 mins, followed by proteinase $\mathrm{K}$ digestion for 3 days at $56^{\circ} \mathrm{C}$. Genomic DNA was then extracted using the DNeasy Tissue kit according to the manufacturer's instructions.

Bisulfite modification. One microgram of genomic DNA was bisulfite modified using the MethylEasy Xceed kit (Human Genetic Signatures, North Ryde, Australia) according to the manufacturer's instructions. Bisulfite modified DNA was eluted twice with $50 \mu \mathrm{L}$ of elution buffer in an estimated concentration of $10 \mathrm{ng} / \mu \mathrm{L}$.

Preparation of methylation standards. Commercially available methylated DNA (Millipore, Billerica, MA) was used as a fully methylated control. To prepare unmethylated DNA, peripheral blood DNA obtained from a healthy individual underwent two rounds of whole genome amplification (WGA) using the Illustra GenomiPhi V2 DNA Amplification Kit (GE Healthcare, Giles, UK) according to the manufacturer's instructions. The first round of WGA was performed with $1 \mathrm{ng}$ of peripheral blood DNA. One microliter of a 10-fold dilution of the first round WGA product was used for the second round of WGA. After bisulfite modification, a qPCR assay that amplified a region lacking $\mathrm{CpG}$ dinucleotides within the COL2A1 gene was performed to quantify the amount of template ${ }^{47}$. A series of methylation dilution standards of $100 \%, 50 \%, 20 \%, 10 \%, 5 \%$ and $1 \%$ were prepared by mixing of the fully methylated DNA with the WGA DNA.

Methylation sensitive-high resolution melting assays. MS-HRM assays were designed either to target the promoter region or to overlap the regions used in previously reported assays. To better work with fragmented FFPE DNA, MS-HRM assays were designed to amplify a short amplicon size of less than $150 \mathrm{bp}$. Primer sequences, amplicon sizes, $\mathrm{MgCl}_{2}$ concentration, annealing temperatures, and the number of $\mathrm{CpG}$ dinucleotides analysed are summarised in Table 3. PCR amplification and MS-HRM were performed on the RotorGene Q (Qiagen). The PCR mixture was prepared in a final volume of $20 \mu \mathrm{L}$ and contained: $10 \mathrm{ng}$ of bisulfite modified template, $1 \times$ PCR buffer, $2.5-4 \mathrm{mM} \mathrm{MgCl}_{2}, 200-400 \mathrm{nM}$ each primer, $200 \mu \mathrm{M}$ dNTPs, $5 \mu \mathrm{M}$ SYTO9 and $0.5 \mathrm{U}$ HotStar Taq (Qiagen). PCR cycling and melting conditions were as follows; one cycle of $95^{\circ} \mathrm{C}$ for 15 minutes; $50-55$ cycles of $95^{\circ} \mathrm{C}$ for 10 seconds, annealing temperature of each assay for 20 seconds, $72^{\circ} \mathrm{C}$ for 25 seconds; one cycle of $97^{\circ} \mathrm{C}$ for one minute and a melt from $70^{\circ} \mathrm{C}$ to $95^{\circ} \mathrm{C}$ rising $0.2^{\circ} \mathrm{C}$ per second. The melting profiles of amplicons were analysed using RotorGene $\mathrm{Q}$ Software (v1.7). All samples were tested in duplicate.

Bisulfite Sanger sequencing. To confirm the MS-HRM results for ERCC1, NEIL1 and RAD23B methylation, MS- HRM products were sequenced using the Big Dye Terminator v3.1 chemistry (Applied Biosystems). Sequencing products were purified with the Agencourt CleanSEQ reagent (Beckman Coulter) and analysed by capillary electrophoresis on an ABI3730 sequencer (Applied Biosystems). The sequencing data was analysed using Sequencher 4.6 (Gene Codes Corporation).
Analysis of DNA methylation from publically available data. Marmal-aid (http:// www.marmal-aid.org) is a data repository of publically available genome-scale DNA methylation analysis using the Illumina HumanMethylation $450 \mathrm{~K}$ (HM450K) beadchip. At the time of download, 8,654 data sets were available from marmal-aid and also contained data from The Cancer Genome Atlas (TCGA) study ${ }^{48}$. At the time of download, a total of 568 non-small cell lung cancers (341 adenocarcinomas and 227 squamous carcinomas) were available from marmal-aid from TCGA. We identified 11 HM450K probes interrogating CpG sites within our MS-HRM amplicons. Raw beta values were extracted using marmal-aid in R. Data were extracted according to their annotations in marmal-aid. Samples annotated with "lung" and "cancer" and our probes of interest were used for downstream analysis and visualization in $\mathrm{R}$ (http://www.cran.org). Using guidelines previously described ${ }^{49}$, a $\beta$-value of greater than 0.2 was used to define the presence of DNA methylation.

1. Taron, M. et al. BRCA1 mRNA expression levels as an indicator of chemoresistance in lung cancer. Hum Mol Genet 13, 2443-9 (2004).

2. Rosell, R. et al. Customized treatment in non-small-cell lung cancer based on EGFR mutations and BRCA1 mRNA expression. PLoS One 4, e5133 (2009).

3. Papadaki, C. et al. ERCC1 and BRAC1 mRNA expression levels in the primary tumor could predict the effectiveness of the second-line cisplatin-based chemotherapy in pretreated patients with metastatic non-small cell lung cancer. J Thorac Oncol 7, 663-71 (2012).

4. Papadaki, C. et al. Correlation of BRCA1, TXR1 and TSP1 mRNA expression with treatment outcome to docetaxel-based first-line chemotherapy in patients with advanced/metastatic non-small-cell lung cancer. Br J Cancer 104, 316-23 (2011).

5. Boukovinas, I. et al. Tumor BRCA1, RRM1 and RRM2 mRNA expression levels and clinical response to first-line gemcitabine plus docetaxel in non- small-cell lung cancer patients. PLoS One 3, e3695 (2008).

6. Rosell, R. et al. BRCA1: a novel prognostic factor in resected non-small-cell lung cancer. PLoS One 2, el129 (2007).

7. Font, A. et al. BRCA1 mRNA expression and outcome to neoadjuvant cisplatinbased chemotherapy in bladder cancer. Ann Oncol 22, 139-44 (2011).

8. Weberpals, J. et al. The DNA repair proteins BRCA1 and ERCC1 as predictive markers in sporadic ovarian cancer. Int J Cancer 124, 806-15 (2009).

9. Dabholkar, M. et al. ERCC1 and ERCC2 expression in malignant tissues from ovarian cancer patients. J Natl Cancer Inst 84, 1512-7 (1992).

10. Middleton, M. R. \& Margison, G. P. Improvement of chemotherapy efficacy by inactivation of a DNA-repair pathway. Lancet Oncol 4, 37-44 (2003).

11. Esteller, M. et al. Inactivation of the DNA-repair gene MGMT and the clinical response of gliomas to alkylating agents. N Engl J Med 343, 1350-4 (2000).

12. Helleday, T., Petermann, E., Lundin, C., Hodgson, B. \& Sharma, R. A. DNA repair pathways as targets for cancer therapy. Nat Rev Cancer 8, 193-204 (2008).

13. Mikeska, T., Bock, C., Do, H. \& Dobrovic, A. DNA methylation biomarkers in cancer: progress towards clinical implementation. Expert Rev Mol Diagn 12, 473-87 (2012).

14. Curtin, N. J. DNA repair dysregulation from cancer driver to therapeutic target. Nat Rev Cancer 12, 801-17 (2012).

15. Mikeska, T., Candiloro, I. \& Dobrovic, A. The implications of heterogeneous DNA methylation for the accurate quantification of methylation. Epigenomics 2, 561-573 (2010)

16. Cottrell, S. E. \& Laird, P. W. Sensitive detection of DNA methylation. Ann N Y Acad Sci 983, 120-30 (2003).

17. Brandes, J. C., Carraway, H. \& Herman, J. G. Optimal primer design using the novel primer design program: MSPprimer provides accurate methylation analysis of the ATM promoter. Oncogene 26, 6229-37 (2007).

18. Wojdacz, T. K. \& Dobrovic, A. Methylation-sensitive high resolution melting (MS-HRM): a new approach for sensitive and high-throughput assessment of methylation. Nucleic Acids Res 35, e41 (2007).

19. Lord, R. V. et al. Low ERCC1 expression correlates with prolonged survival after cisplatin plus gemcitabine chemotherapy in non-small cell lung cancer. Clin Cancer Res 8, 2286-91 (2002).

20. Huang, K. T. et al. DNA methylation profiling of phyllodes and fibroadenoma tumours of the breast. Breast Cancer Res Treat 124, 555-65 (2010).

21. Wong, E. M. et al. Constitutional methylation of the BRCA1 promoter is specifically associated with BRCA1 mutation-associated pathology in early- onset breast cancer. Cancer Prev Res (Phila) 4, 23-33 (2011).

22. Mikeska, T., Carney, D. A., Seymour, J. F. \& Dobrovic, A. No evidence for DNA methylation of the ATM promoter $\mathrm{CpG}$ island in chronic lymphocytic leukemia. Leuk Lymphoma 53, 1420-2 (2012).

23. $\mathrm{Wu}, \mathrm{Y}$. H. et al. Xeroderma pigmentosum group $\mathrm{C}$ gene expression is predominantly regulated by promoter hypermethylation and contributes to p53 mutation in lung cancers. Oncogene 26, 4761-73 (2007).

24. Chaisaingmongkol, J. et al. Epigenetic screen of human DNA repair genes identifies aberrant promoter methylation of NEIL1 in head and neck squamous cell carcinoma. Oncogene 31, 5108-16 (2012).

25. Chen, H. Y., Shao, C. J., Chen, F. R., Kwan, A. L. \& Chen, Z. P. Role of ERCC1 promoter hypermethylation in drug resistance to cisplatin in human gliomas. Int $J$ Cancer 126, 1944-54 (2010).

26. Peng, B. et al. Epigenetic silencing of the human nucleotide excision repair gene, hHR23B, in interleukin-6-responsive multiple myeloma KAS-6/1 cells. J Biol Chem 280, 4182-7 (2005). 
27. Virmani, A. K. et al. Aberrant methylation of the adenomatous polyposis coli (APC) gene promoter 1A in breast and lung carcinomas. Clin Cancer Res 7, 1998-2004 (2001)

28. Brock, M. V. et al. DNA methylation markers and early recurrence in stage I lung cancer. N Engl J Med 358, 1118-28 (2008).

29. Vaissiere, T. et al. Quantitative analysis of DNA methylation profiles in lung cancer identifies aberrant DNA methylation of specific genes and its association with gender and cancer risk factors. Cancer Res 69, 243-52 (2009).

30. Hegi, M. E. et al. MGMT gene silencing and benefit from temozolomide in glioblastoma. N Engl J Med 352, 997-1003 (2005).

31. Hegi, M. E., Sciuscio, D., Murat, A., Levivier, M. \& Stupp, R. Epigenetic deregulation of DNA repair and its potential for therapy. Clin Cancer Res 15, 5026-31 (2009).

32. Tomii, K. et al. Aberrant promoter methylation of insulin-like growth factor binding protein-3 gene in human cancers. Int J Cancer 120, 566-73 (2007).

33. Das, P. M. et al. Methylation mediated silencing of TMS1/ASC gene in prostate cancer. Mol Cancer 5, 28 (2006).

34. Enokida, H. et al. Ethnic group-related differences in CpG hypermethylation of the GSTP1 gene promoter among African-American, Caucasian and Asian patients with prostate cancer. Int J Cancer 116, 174-81 (2005).

35. Herman, J. G., Graff, J. R., Myohanen, S., Nelkin, B. D. \& Baylin, S. B. Methylationspecific PCR: a novel PCR assay for methylation status of CpG islands. Proc Natl Acad Sci U S A 93, 9821-6 (1996).

36. Chiang, J. W., Karlan, B. Y., Cass, L. \& Baldwin, R. L. BRCA1 promoter methylation predicts adverse ovarian cancer prognosis. Gynecol Oncol 101, 403-10 (2006).

37. Dobrovic, A. \& Simpfendorfer, D. Methylation of the BRCA1 gene in sporadic breast cancer. Cancer Res 57, 3347-50 (1997).

38. Marsit, C. J. et al. Inactivation of the Fanconi anemia/BRCA pathway in lung and oral cancers: implications for treatment and survival. Oncogene 23, 1000-4 (2004).

39. Lee, M. N. et al. Epigenetic inactivation of the chromosomal stability control genes BRCA1, BRCA2, and XRCC5 in non-small cell lung cancer. Clin Cancer Res 13, 832-8 (2007).

40. van Dijk, J. P. et al. A novel, essential control for clonality analysis with human androgen receptor gene polymerase chain reaction. Am J Pathol 161, 807-12 (2002).

41. Kupper, D., Reuter, M., Meisel, A. \& Kruger, D. H. Reliable detection of DNA CpG methylation profiles by the isoschizomers MspI/HpaII using oligonucleotide stimulators. Biotechniques 23, 843-7 (1997).

42. Nouspikel, T. DNA repair in mammalian cells: Nucleotide excision repair: variations on versatility. Cell Mol Life Sci 66, 994-1009 (2009).

43. Khan, O. et al. HR23B is a biomarker for tumor sensitivity to HDAC inhibitorbased therapy. Proc Natl Acad Sci U S A 107, 6532-7 (2010).

44. Miyanaga, A. et al. Antitumor activity of histone deacetylase inhibitors in nonsmall cell lung cancer cells: development of a molecular predictive model. $\mathrm{Mol}$ Cancer Ther 7, 1923-30 (2008)

45. Bandaru, V., Sunkara, S., Wallace, S. S. \& Bond, J. P. A novel human DNA glycosylase that removes oxidative DNA damage and is homologous to Escherichia coli endonuclease VIII. DNA Repair (Amst) 1, 517-29 (2002).

46. Rosenquist, T. A. et al. The novel DNA glycosylase, NEIL1, protects mammalian cells from radiation-mediated cell death. DNA Repair (Amst) 2, 581-91 (2003).

47. Virmani, A. K. et al. Hierarchical clustering of lung cancer cell lines using DNA methylation markers. Cancer Epidemiol Biomarkers Prev 11, 291-7 (2002).

48. Cancer Genome Atlas Research, N. Comprehensive genomic characterization of squamous cell lung cancers. Nature 489, 519-25 (2012).

49. Bibikova, M. et al. Genome-wide DNA methylation profiling using Infinium(R) assay. Epigenomics 1, 177-200 (2009).
50. Safar, A. M. et al. Methylation profiling of archived non-small cell lung cancer: a promising prognostic system. Clin Cancer Res 11, 4400-5 (2005)

51. Safar, A. M. et al. Promoter hypermethylation for molecular nodal staging in nonsmall cell lung cancer. Arch Pathol Lab Med 131, 936-41 (2007).

52. Wang, Y. et al. Multiple gene methylation of nonsmall cell lung cancers evaluated with 3-dimensional microarray. Cancer 112, 1325-36 (2008).

53. Wu, J. Y. et al. Association of O6-methylguanine-DNA methyltransferase (MGMT) promoter methylation with p53 mutation occurrence in non-small cell lung cancer with different histology, gender, and smoking status. Ann Surg Oncol 15, 3272-7 (2008).

54. Feng, Q. et al. DNA methylation in tumor and matched normal tissues from nonsmall cell lung cancer patients. Cancer Epidemiol Biomarkers Prev 17, 645-54 (2008).

55. Liu, Y., Lan, Q., Siegfried, J. M., Luketich, J. D. \& Keohavong, P. Aberrant promoter methylation of p16 and MGMT genes in lung tumors from smoking and never-smoking lung cancer patients. Neoplasia 8, 46-51 (2006).

56. Kim, Y. T. et al. Prognostic implication of aberrant promoter hypermethylation of CpG islands in adenocarcinoma of the lung. J Thorac Cardiovasc Surg 130, 1378 (2005).

57. Tang, M., Torres-Lanzas, J., Lopez-Rios, F., Esteller, M. \& Sanchez-Cespedes, M. Wnt signaling promoter hypermethylation distinguishes lung primary adenocarcinomas from colorectal metastasis to the lung. Int J Cancer 119, 2603-6 (2006).

58. Seng, T. J. et al. DLEC1 and MLH1 promoter methylation are associated with poor prognosis in non-small cell lung carcinoma. Br J Cancer 99, 375-82 (2008).

59. Wang, Y. C. et al. Inactivation of hMLH1 and hMSH2 by promoter methylation in primary non-small cell lung tumors and matched sputum samples. J Clin Invest 111, 887-95 (2003)

60. Geng, X., Wang, F., Zhang, L. \& Zhang, W. M. Loss of heterozygosity combined with promoter hypermethylation, the main mechanism of human MutL Homolog (hMLH1) gene inactivation in non-small cell lung cancer in a Chinese population. Tumori 95, 488-94 (2009).

\section{Acknowledgments}

We would like to thank Thomas Mikeska for critical discussion. This project was supported by funding from Cancer Australia to A.D., the Cancer Council of Victoria to A.D., and the National Health and Medical Research Council of Australia to P.M. and A.D. H.D. is recipient of Postdoctoral Fellowship from the Cancer Council of Victoria.

\section{Author contributions}

Suitable samples were identified by P.M., T.J., B.S. and C.M. The experimental work was carried out by H.D. and the TCGA data was analysed by N.W., H.D. and N.W. prepared figures. H.D. and A.D. co-wrote the manuscript. All authors approved the final manuscript

\section{Additional information}

Competing financial interests: The authors declare no competing financial interests.

How to cite this article: Do, H. et al. A critical re-assessment of DNA repair gene promoter methylation in non-small cell lung carcinoma. Sci. Rep. 4, 4186; DOI:10.1038/srep04186 (2014).

\footnotetext{
(c) (i) (2) This work is licensed under a Creative Commons AttributionBY NC SA NonCommercial-ShareAlike 3.0 Unported license. To view a copy of this license,
} visit http://creativecommons.org/licenses/by-nc-sa/3.0 